\title{
La prevalencia de comportamientos agresivos en el hospital, en la familia y en las residencias comunitarias
}

\author{
Man Cheung Chung* \\ Stuart Cumella** \\ Wai-Ling Bickerton*** \\ Caroline Winchester** \\ * Universidad de Plymouth \\ ** Universidad de Birmingham \\ *** Universidad de Southampton \\ REINO UNIDO
}

\begin{abstract}
RESUMEN - Este estudio piloto describe la prevalencia de comportamientos agresivos en pacientes con discapacidades en el aprendizaje ( $\mathrm{N}=98$ ) que viven en tres diferentes lugares (hospitales, familias y residencias comunitarias) en un distrito de salud inglés. Se entrevistó a noventa y ocho cuidadores teniendo en cuenta el perfil clínico de los enfermos, utilizando para ello una Escala de Valoración de la Discapacidad, una lista para chequear el comportamiento aberrante y un inventario psicopatológico para adultos con retraso mental. Los resultados mostraron que las discapacidades de nuestros enfermos en las tres modalidades de residencia eran similares, a lo que hay que añadir algunas diferencias significativas en cuanto a la visión, lectura, escritura y en las habilidades de cálculo. Los comportamientos agresivos no pasaban de ser un problema leve no encontrándose diferencias importantes en cuanto al tipo de residencia. Otros muchos análisis del comportamiento agresivo que suponían un problema en su no mas que leve fueron tratados. Todos los enfermos podrían ser diagnosticados como de padecer de adaptaciones defectuosas. Los coeficientes de correlación mostraron importantes relaciones entre el comportamiento agresivo y la psicopatología presente en los tres modalidades de residencia, especialmente entre los enfermos que vivían en comunidad.
\end{abstract}

\section{Introducción}

Este estudio surgió a partir de la fusión de tres distritos de salud ingleses en uno solo y ocurrió en una ciudad inglesa en la que se constituyó el nuevo distrito de salud. Esta nueva estructura hizo necesaria la preparación de una estrategia que se adaptase a las necesidades de los pacientes con discapaci- 
dades en el aprendizaje. Para lograrlo, se necesitaba contar con una buena estimación de las necesidades en cuanto a cuidados de la salud en el nuevo distrito. Por esta razón, los investigadores iniciaron su estudio centrándose en la estimación de la prevalencia de los comportamientos agresivos y los aspectos psicopatológicos entre los individuos con discapacidades en el aprendizaje.

El término comportamiento agresivo o mejor "desafiante" se utiliza para designar a aquellos individuos con una discapacidad en el aprendizaje cuyo comportamiento provoca problemas de dirección en aquellos lugares donde residen (salas de hospitales, hostales). Estos comportamientos incluyen actos de violencia irracional y destructivos de cosas, o actos agresivos contra sí mismo o contra los demás. Los individuos con comportamientos agresivos o desafiantes pueden presentar conductas como escupir, difamar, provocarse vómitos, pica, comer y beber continuamente, así como blasfemar o tener comportamientos sexuales inapropiados. Pueden también acumular suciedad, abandonarse en sus cuidados corporales e higiénicos o adoptar comportamientos estereotipados. Del mismo modo, cabe la posibilidad de que pudiesen también hacer ruidos molestos, insultar o lanzar objetos y crear en definitiva situaciones de desorden y caos.

Algunas investigaciones han mostrado que aproximadamente el $47,8 \%$ de los enfermos con problemas de aprendizaje adoptan comportamientos agresivos (Lakin et al. 1993), lo que parece ser una situación más frecuente en entornos institucionales (Baumeister \& Rollings 1976, Fovel et al. 1989, Griffin et al. 1986, Maurice \& Trudel 1982, Schroeder et al. 1978) que en aquellos que no lo eran (Hills \& Bruininks 1984, Rojahn 1996). Borthwick-Duffy (1994) afirmó que existían más comportamientos agresivos, como por ejemplo hacer daño a los demás (el 13\%), lesionarse a sí mismo (el 12\%) y destruir propiedades (el 30\%) en instituciones que en el hogar familiar $(0,8 \%$ - 3,2\%), casas independientes $(0,7 \%$ $1,7 \%)$, hogares "protegidos" de la comunidad, centros de salud de la comunidad $(2,2 \%-9,2 \%)$ y "nursing homes" $(2,2 \%$ $11,2 \%)$. Además, la investigación sobre los gastos médicos a nivel nacional del año 1987 (NMES), ya mostraba como el porcentaje de individuos que adoptaban comportamientos agresivos era más alto en los lugares en los que se daba una mayor densidad de población que en los que había menor densidad (en el primer caso variaba entre un $16 \%$ y un $30 \%$ y en el segundo entre el $15 \%$ y el 25\%, Lakin et al. 1989). Bruininks et al. (1994) también descubrieron que el porcentaje de autolesiones en instituciones era de un $47 \%$, mientras que en los hogares familiares, conjuntos de hogares, hogares de acogida y hogares semi-independientes era de entre un $10 \%$ a un $30 \%$. El predominio de las agresiones a terceros en instituciones era casi de un $50 \%$ comparado con el intervalo de un $14 \%$ y hasta de un $36 \%$ en otros hogares. El predominio de destrucción de la propiedad en las instituciones rondaba el $34 \%$, mientras que el intervalo variaba de un $10 \%$ a casi un $30 \%$ en otros hogares.

Qureshi y Alborz (1992), una vez hubieron estudiado a 4.200 individuos correspondientes a siete áreas de salud, se encontraron con que el 30\% de los que vivían en hospitales y el $13 \%$ de aquellos que vivían en comunidad mostraban comportamientos agresivos. Además, Qureshi (1994) descubrió que aproximadamente un $14 \%$ de los individuos que vivían en hospitales mostraban comportamientos agresivos. Tan sólo un 5\% de los que vivían en comunidad mostraban comportamientos agresivos. Otro estudio llevado a cabo en instituciones de larga estancia, mostraba unos porcentajes similares de entre 
un $8 \%$ a un $15 \%$ (Murphy 1985), correspondiendo el porcentaje más bajo a los hogares familiares $(1,1 \%)$, las agrupaciones de hogares $(2,7 \%)$, y los hogares residenciales (10,8\%) (Kebbon \& Windahl 1985).

Centrándonos en el comportamiento agresivo y en la forma de comportamiento autoagresivo, diversos estudios han mostrado como individuos con dificultades de aprendizaje que viven en instituciones muestran más actitudes agresivas que el resto. Oliver et al. (1987), después de haber estudiado a 596 personas, confirmó que el $51 \%$ de aquellos que habían sido examinados en hospitales, el $28 \%$ de los que estaban en residencias y el $21 \%$ de los hogares familiares presentaban comportamientos de tipo agresivo. Emerson (1992) aportaba un porcentaje aún más bajo (de un 10\% a un 15\%) en hospitales, la mitad de los cuales también mostraban otros tipos de comportamientos conflictivos. Según Hill y Bruininks (1984), un 11,1\% de los individuos residentes en comunidad adoptaron un comportamiento autolesivo, mientras que un 17,6\% lo tenían en residencias públicas. Rojahn (1986) por su parte, mostraba la existencia de menores porcentajes de autolesión $(1,7 \%)$ entre los individuos que residían en comunidad. Centrándonos en este tipo de comportamientos agresivos, Russell y Harris (1993) descubrieron, en un estudio realizado sobre 370.000 individuos, que un $55,4 \%$ de los pacientes hospitalizados, un $25 \%$ de los que convivían en familias y un 17,3\% de los que vivían en alojamientos para el personal o en residencias, al menos una vez habían llevado a cabo una agresión física.

Por supuesto, no todos los estudios han confirmado que el comportamiento agresivo tienda a ser menor en lugares no institucionales. Por ejemplo, Kernan y Quershi (1993), después de realizar un estudio en 734 individuos con problemas de aprendizaje, tanto en hospitales de larga estancia (el 30\%) como en comunidades (el 13\%), descubrieron que la proporción era casi igual para ambos tipos de residencias (hospital $=$ el 37,4\%; comunidad $=$ el 37,7\%). Clarke et al. (1990) realizó un estudio similar que se centraba en aquellos individuos que adoptaban cualquier "comportamiento agresivo específico". Con esta amplia definición, se encontraron con que la diferencia entre las muestras institucionales y las no institucionales era pequeña (el $42 \%$ en hospitales, el $36 \%$ en comunidades y el $41 \%$ en hogares familiares). Sin embargo, de momento, se ha aceptado de forma general que los pacientes se adaptan mejor a los centros privados que a los públicos, al menos en lo que se refiere a la mejora de su capacidad de adaptación (Larson \& Lakin 1989) y a la participación social y comunitaria (Molony \& Taplin 1988, Conroy \& Bradley 1985, Conroy et al. 1991, Horner et al. 1988).

Se han hecho intentos con el fin de identificar la relación existente entre los comportamientos desafiantes y agresivos y las enfermedades psiquiátricas. Pues bien, los individuos con discapacidades en el aprendizaje y enfermedad mental, corren un mayor riesgo de desarrollar comportamientos agresivos (Borthwick-Duffy 1990, Borthwick-Duffy \& Eyman 1990, Bruininks et al. 1988). Borthwick-Duffy (1994) explica que el $8,5 \%$ de los individuos con cólera agresiva, el 5,9\% de los que adoptaban comportamientos autolesivos y el $19,4 \%$ de los que tenían conductas destructivas de bienes, tenían un diagnóstico dual (discapacidad en el aprendizaje y trastorno psiquiátrico). Otros estudios también señalan como el $12 \%$ de sus muestras comprendían diagnósticos de depresión, trastornos afectivos, esquizofrenia y otras patologías psicóticas inclasificables (Kiernan \& Qureshi 1993). 


\section{Método}

La población sometida a esta investigación fue tomada del Registro de Discapacidad de la ciudad. Se componía de individuos con discapacidades de aprendizaje, con más de 16 años y que residían en uno de los tres distritos de salud. Un total de 1.409 nombres fueron identificados y distribuidos entre cada uno de los tres distritos (Distrito A: $N=561$, Distrito B: $N=339, C: N=509$ ). Sin embargo, las autoridades de uno de los tres distritos no nos concedió permiso para contactar con los enfermos que vivían en él. La muestra por tanto al final quedó formada por 900 individuos de los dos distritos restantes. Con el fin de llevar a cabo una valoración detallada se seleccionó a un 14\% de una muestra cuasi aleatoria de 126 personas. Veintiocho de ellas se excluyeron con posterioridad porque habían cambiado de dirección, porque habían muerto o porque se habían mudado a otra ciudad. Finalmente quedaron 98 individuos con un porcentaje de respuesta de un $77 \%$.

Los cuidadores del enfermo (familiares $\mathrm{u}$ otros empleados en estas labores) de los 98 enfermos fueron entrevistados, utilizando para ello el Programa de Valoración de la Incapacidad (DAS), la Lista de Comprobación de los Comportamientos Aberrantes (ABC) y el Inventario de Psicopatología para Adultos con Retraso Mental (PIMRA). Todos los entrevistadores habían sido entrenados en el uso de estos instrumentos de investigación y contaban con la experiencia de haber trabajado con personas con problemas de discapacidad en el aprendizaje. La definición de comportamiento desafiante o agresivo de la cual hacemos uso en este estudio, hace referencia a que estos comportamientos de los pacientes causaban problemas en la vida diaria de las instituciones en donde residían.

\section{Medidas}

El Escala de Valoración de la Incapacidad (DAS) recoge información acerca de discapacidades psíquicas, habilidades, interacción social y anormalidades en el comportamiento. El DAS se diseñó inicialmente para valorar a aquellos enfermos reubicados procedentes de un hospital y con discapacidades en el aprendizaje. Más tarde incorporó una versión mas amplia de escalas del Equipo de Desarrollo Nacional Wessex así como de los ítems procedentes de la MRC Handicaps, Behaviour and Skills Schedule (Wing 1989).

La Lista de Comprobación de Comportamientos Aberrantes (ABC) es un instrumento psicométrico de 58 ítems, que mide los comportamientos agresivos asociados a discapacidades en el aprendizaje. Consiste en una escala de cuatro puntos $(0=$ sin problema alguno, 1 = problema leve, $2=$ problema moderadamente importante, $3=$ problema grave). Los 58 ítems se descomponen en cinco factores (subescalas): irritabilidad, agitación y llanto (Factor I, 15 ítems, puntuación máxima $=45)$; letargia y extroversión social (Factor II, 16 ítems, puntuación máxima $=48)$; comportamiento estereotipado (Factor III, 7 ítems, puntuación máxima = 21); hiperactividad y desobediencia (Factor IV, 16 ítems, máxima puntuación = 48) y habla inapropiada (Factor V, 4 ítems, puntuación máxima = 12) (Aman, et al. 1985a; Aman et al. 1985b).

El Inventario de Psicopatolgía para adultos con Retraso Mental (PIMRA) consiste en una lista de comportamientos psicopatológicos que se emplea en individuos con dificultades en el aprendizaje y con enfermedad mental. Estudia los 8 síntomas principales de la esquizofrenia, trastorno afectivo, desorden psicosexual, trastorno de adapta- 
ción, trastorno de ansiedad, trastorno somatoforme, trastorno de personalidad y adaptaciones inapropiadas, cada uno de los cuales deriva de 7 ítems. Con el fin de confirmar el diagnóstico psiquiátrico, los individuos debían al menos tachar 4 de los 7 ítems (Matson 1988).

\section{Resultados}

La media de edad de los 98 individuos (49 mujeres y 49 hombres respectivamente) era de 39 años, dentro de un intervalo que iba de 18 a 76 años. Por entonces, 12 vivían en hospitales, 38 con sus familias, y 48 vivían en casas residenciales de la comunidad. Los datos demográficos de estos individuos según los tres lugares de residencia pueden verse en la tabla I. La edad de los residentes en hospitales era mayor que la de los otros dos grupos, especialmente aquellos que residían en familia. La distribución por sexos era prácticamente igual.

La puntuación media de los ítems DAS para los tres grupos se resumen en la tabla II. Mediante el ANOVA de una vía y el test Turkey-HSD se determinó si los tres grupos difieren entre ellos. En general, la incapacidad de los individuos en cada uno de los tres grupos era tan parecida que apenas había diferencia en la mayoría de los ítems. Los enfermos que residían en hospitales tenían mucha menor movilidad (42\%) que los demás $(70 \%)$. Aproximadamente un $70 \%$ del total eran continentes mientras que los que vivían en comunidad eran relativamente menos. Además, de entre estos, más del $80 \%$ del total podía ir por sí mismo al servicio. Los enfermos residentes en hospitales, y la mayoría de los que vivían en comunidad y en familias $(80 \%)$ comían por si mismos. Los residentes en la comunidad tenían una mayor capacidad para lavarse y vestirse que lo que sucedía en el resto de los grupos (menos de un 30\%).

Todos los enfermos residentes en hospitales y mas del $80 \%$ de los que residentes en comunidades tenían capacidades visuales y auditivas normales. Se daba una diferencia importante en cuanto a la visión ( $\mathrm{df}=2, \mathrm{~F}=$ $3,36, \mathrm{p}<, 038$ ) entre los enfermos que vivían en familias (66\%) y los de los otros dos grupos, en especial en aquellos que residían en los hospitales. Prácticamente un $75 \%$ de éstos y aproximadamente la mitad de los pertenecientes a los otros dos grupos eran capaces de hacerse entender hablando. Sin embargo, estos dos grupos tenían menos capacidad para comprender que los que residían en hospitales.

La escritura, la lectura y las habilidades de cálculo eran limitadas (menos de un $25 \%$ ). Los residentes en hospitales tenían mayor limitación en la lectura $(\mathrm{df}=2, \mathrm{~F}=$ $3,36, \mathrm{p}<, 038)$ y escritura $(\mathrm{df}=2, \mathrm{~F}=$ $3,29, \mathrm{p}<, 041)$ que los otros dos grupos.

Tabla I

Datos demográficos de los pacientes en los tres grupos

Características

Familia

$$
\mathrm{N}=38
$$

Hospital

$\mathrm{N}=12$
Residencias en la comunidad $\mathrm{N}=48$

\begin{tabular}{lccc}
\hline Años edad & $28,65(\mathrm{sd}=9,98)$ & $54,08(\mathrm{sd}=14,60)$ & $42,54(\mathrm{sd}=14,39)$ \\
Rango & $18-62$ & $34-76$ & $18-66$ \\
Hombre & 17 & 7 & 25 \\
Mujer & 21 & 5 & 23 \\
\hline
\end{tabular}


Los residentes en hospitales no disponían de habilidades de cálculo, mientras que para los otros dos grupos, sobre todos los que vivían en comunidad, éste era un rasgo que les caracterizaba $(\mathrm{df}=2, \mathrm{~F}=$ $4,68, \mathrm{p}<, 011)$. Las habilidades domésticas, manuales y ocupacionales eran un tanto limitadas, sobre todo en los dos últimos grupos mencionados (menos de un $10 \%)$. Los enfermos residentes en la comunidad y en los hospitales eran sociables mientras que, en familias, tan sólo el $30 \%$ era sociable.
Los resultados de los cinco factores del ABC aparecen en la tabla III. Muestran cómo la media de la puntuación disminuye entre las escalas de "sin problema" y "un ligero problema”. La ANOVA de una vía no mostró grandes diferencias entre los grupos, a pesar de que existía una tendencia a que los comportamientos agresivos de los enfermos residentes en hospitales fuesen de menor gravedad que los de los otros dos grupos. Sin embargo, en general el comportamiento agresivo era prácticamente similar para todos, perteneciesen a un grupo o a otro.

Tabla II

Puntuación media de la Escala de Valoración de la discapacidad en los tres grupos

\begin{tabular}{|c|c|c|c|c|c|c|}
\hline & \multicolumn{2}{|c|}{ Familia } & \multicolumn{2}{|c|}{ Hospital } & \multicolumn{2}{|c|}{$\begin{array}{c}\text { Residencia } \\
\text { en la comunidad }\end{array}$} \\
\hline & Media & SD & Media & $\mathrm{SD}$ & Media & SD \\
\hline Movilidad & 4,09 & 1,44 & 3,50 & 1,56 & 4,33 & 1,22 \\
\hline Continencia & 5,00 & 1,59 & 4,25 & 0,75 & 4,08 & 1,38 \\
\hline Aseo personal & 2,71 & 0,69 & 3,00 & 0,00 & 2,62 & 0,81 \\
\hline Alimentación & 2,86 & 0,34 & 3,00 & 0,00 & 2,72 & 0,61 \\
\hline Lavarse & 2,15 & 0,54 & 2,08 & 0,51 & 2,37 & 0,70 \\
\hline Vestirse & 2,63 & 0,94 & 2,50 & 0,90 & 2,85 & 1,11 \\
\hline Visión & 2,60 & 0,59 & 3,00 & 0,00 & 2,79 & 0,45 \\
\hline Audición & 2,81 & 0,45 & 3,00 & 0,00 & 2,95 & 0,28 \\
\hline Hacerse entender & 4,15 & 1,07 & 4,58 & 0,90 & 4,20 & 1,14 \\
\hline Comprender la información & 4,10 & 1,08 & 4,16 & 0,71 & 4,08 & 1,18 \\
\hline Lectura & 2,73 & 1,91 & 1,16 & 0,38 & 2,75 & 2,22 \\
\hline Escritura & 2,57 & 1,84 & 1,08 & 0,28 & 2,56 & 2,11 \\
\hline Cálculo & 1,63 & 0,63 & 1,00 & 0,00 & 1,72 & 0,89 \\
\hline Habilidad doméstica & 2,18 & 0,83 & 2,16 & 0,83 & 2,33 & 0,83 \\
\hline Manualidades & 1,76 & 1,21 & 1,33 & 1,23 & 1,58 & 1,28 \\
\hline Habilidad laboral & 0,28 & 0,86 & 0,25 & 0,86 & 0,22 & 0,77 \\
\hline Sociabilidad & 5,34 & 2,68 & 5,50 & 2,90 & 6,00 & 2,49 \\
\hline
\end{tabular}

Tabla III

Puntuación media de la lista de Conductas Aberrantes en los tres grupos

\begin{tabular}{lcccccc} 
& \multicolumn{2}{c}{ Familia } & \multicolumn{2}{c}{ Hospital } & \multicolumn{2}{c}{$\begin{array}{c}\text { Residencias } \\
\text { en la comunidad } \\
\text { Media }\end{array}$} \\
& Media & SD & Media & SD & & Med \\
\hline Irritabilidad & 8,31 & 7,08 & 5,41 & 4,25 & 9,02 & 9,00 \\
Letargia & 6,52 & 7,03 & 3,75 & 4,39 & 6,08 & 6,52 \\
Estereotipos & 2,63 & 3,30 & 0,58 & 0,99 & 1,81 & 2,81 \\
Hiperactividad & 7,28 & 7,37 & 6,75 & 6,18 & 8,16 & 7,54 \\
Habla inapropiada & 2,42 & 2,91 & 2,25 & 2,83 & 2,39 & 3,33 \\
\hline
\end{tabular}


Los resultados de PIMRA para los tres grupos de enfermos aparecen resumidos en la tabla IV. Muestran como los enfermos pertenecientes a cada uno de los tres grupos se puede considerar que cualquier enfermo de cualquiera de los tres grupos no se ha adaptado satisfactoriamente. Los tests ANOVA de una vía y posteriormente el Tukey-HSD muestran que los enfermos residentes en familia tienen mas probabilidad de recibir un diagnóstico de esquizofrenia que aquellos otros que viven en la comunidad (df $=2, F=3,18, p<, 045)$. A esto hay que añadir que los residentes en núcleos familiares acusaban una mayor tendencia a padecer trastornos de ansiedad que los residentes en hospitales ( $d f=2, F=3,71, p<, 028)$.

Se calcularon los coeficientes de correlación con el fin de identificar la relación entre comportamientos agresivos y la psicopatología en función del lugar de residencia. En cuanto a los enfermos residentes en familia, se estableció una relación entre la hiperactividad y la irritabilidad con los trastornos afectivos $(\mathrm{r}=0,38, \mathrm{p}<, 016$ y $\mathrm{r}=$ $0,60, \mathrm{p}<, 001$ respectivamente) y con los problemas de adaptación $(\mathrm{r}=0,34, \mathrm{p}<, 033$ y $\mathrm{r}=0,54, \mathrm{p}<, 001$ respectivamente). Al letargo se le correlacionó con una adaptación irregular $(\mathrm{r}=-39, \mathrm{p}<, 013)$.

En lo que respecta a los enfermos que provenían de hospitales, la hiperactividad estaba correlacionada con el trastorno somatoforme $(r=0,79, p<, 002)$ y con el trastorno de personalidad $(\mathrm{r}=0,70, \mathrm{p}<$ ,010). La irritabilidad se encontraba correlacionada con el trastorno de personalidad ( $\mathrm{r}$ $=0,62, \mathrm{p}<, 031)$. La letargia estaba relacionado con la adaptación inapropiada $(r=-73$, $\mathrm{p}<, 006)$. El habla inapropiada estaba correlacionada con el trastorno somatoforme $(\mathrm{r}=$ $0,72, \mathrm{p}<, 008)$ y con el trastorno de personalidad $(\mathrm{r}=0,79, \mathrm{p}<, 002)$.

En relación a los enfermos residentes en hogares de la comunidad, los cinco comportamientos agresivos se correlacionaban de una manera u otra, con la psicopatología. La irritabilidad correlacionaba con la esquizofrenia $(r=, 30, p<, 036)$, el trastorno afectivo $(\mathrm{r}=, 55, \mathrm{p}<, 001)$, los problemas de adaptación $(\mathrm{r}=0,49, \mathrm{p}<, 001)$ y el trastorno somatoforme $(r=0,30, p<, 035)$. La letargia estaba correlacionado con la esquizofrenia $(\mathrm{r}=, 38, \mathrm{p}<, 006)$ y el trastorno afectivo $(\mathrm{r}=, 42, \mathrm{p}<, 003)$. El comportamiento este-

Tabla IV

Media de la puntuación del Inventario Psicopatológico para adultos retrasados mentales en los tres grupos

\begin{tabular}{lcccccc} 
& \multicolumn{2}{c}{ Familia } & \multicolumn{2}{c}{ Hospital } & \multicolumn{2}{c}{$\begin{array}{c}\text { Residencias } \\
\text { en la comunidad }\end{array}$} \\
& Media & SD & Media & SD & Media & SD \\
\hline Esquizofrenia & 1,63 & 1,19 & 1,25 & 0,96 & 1,02 & 1,08 \\
Trastorno afectivo & 2,18 & 1,35 & 1,33 & 1,15 & 2,00 & 1,53 \\
Trastorno psicosexual & 0,13 & 0,52 & 0,16 & 0,38 & 0,25 & 0,56 \\
Trastorno de adaptación & 1,73 & 1,22 & 1,66 & 1,23 & 1,87 & 1,45 \\
Trastorno de ansiedad & 2,94 & 1,67 & 1,66 & 1,23 & 2,31 & 1,49 \\
Trastorno somatoforme & 0,55 & 1,00 & 0,33 & 0,88 & 0,85 & 1,41 \\
Trastorno de pesonalidad & 1,47 & 1,37 & 1,00 & 1,20 & 1,72 & 1,14 \\
Adaptación inapropiada & 5,13 & 1,41 & 5,66 & 1,15 & 5,25 & 1,57 \\
\hline
\end{tabular}


reotipado estaba correlacionado con la esquizofrenia $(r=, 40, p<, 004)$ y el trastorno afectivo $(r=0,35, p<, 014)$. La hiperactividad correlacionaba con la esquizofrenia $(\mathrm{r}=, 32, \mathrm{p}<, 024)$ y el trastorno de adaptación $(\mathrm{r}=33, \mathrm{p}<, 019)$. El habla inapropiada se encontraba también correlacionada con la esquizofrenia $(r=, 38, p<, 007)$.

\section{Discusión}

Los resultados de este estudio difieren de los anteriores en el hecho de que el comportamiento agresivo de los enfermos residentes en instituciones (hospitales) y en lugares no institucionales (comunidades y familias) era similar. Su comportamiento sólo causaba problemas poco importantes a la dirección de aquellos lugares en donde vivían. Es muy importante tener en cuenta que la interpretación de nuestros hallazgos precisa de mucha precaución. No debemos pensar que la estimación del predominio que se ha llevado cabo representa la verdadera tendencia de los individuos que mantienen comportamientos agresivos en un área determinada. Existen diferentes razones para hacer esta afirmación: La primera de ellas es que estas estimaciones están sujetas a la complejidad clínica o a la naturaleza cambiante de la conducta de los pacientes. Un estudio longitudinal del comportamiento autolesivo, por ejemplo, mostraba importantes cambios en la población estudiada (Schroeder et al. 1978) en un periodo de tres años.

De hecho, una investigación reciente sobre la evaluación del servicio indica que existe un comportamiento inestable opuesto a un comportamiento mantenido. Por ejemplo, mientras que el comportamiento agresivo disminuía durante la intervención, aumentaba durante el seguimiento (Hoef- kens \& Allen 1990). Si bien tras haber abandonado un centro mayor, el comportamiento agresivo aumentaba al ingresar al paciente en unidades residenciales (Felce et al. 1994) y en alojamientos subvencionados desde el hospital (Lowe et al. 1993), éste disminuía en los enfermos que vivían en ciertas unidades residenciales y en pequeños grupos (Clare \& Murphy 1993, Conneally 1992). $\mathrm{Ni}$ antes ni después de la reubicación, los enfermos no mostraron mejorías en cuanto al predominio de comportamientos agresivos (Wing 1989, Mansell et al. 1992). Incluso en casos donde se daban mejoras importantes, resultaba difícil su mantenimiento (Mansell \& Beasley 1993).

Con referencia al control de los diferentes niveles de capacidad de comunicación que podrían ser la causa de comportamientos agresivos, ya mostramos en otra ocasión que aquellos enfermos que poseían habilidades de comunicación pre-verbal, no mejoraban sus comportamientos agresivos tras seis meses, e incluso un año, de su seguimiento, una vez habían sido trasladados desde un gran hospital a los pequeños pisos residenciales (Chamberlain et al. 1993, Chung et al. 1995). Todos estos hallazgos inconsistentes muestran como los comportamientos agresivos no son tan continuos o estables como pensábamos, aparte de que a menudo están presentes a lo largo del tiempo.

En segundo lugar, a cerca del estudio de los comportamientos desafiantes, a menudo nos encontramos ante la dificultad de definir la naturaleza de estas conductas, como Qureshi (1994) decía “....el término 'comportamiento desafiante o agresivo' está socialmente definido. La gente tiene ideas diferentes sobre lo que significa "desafiante", y de hecho tanto los directores de los hospitales como el personal de las residencias o los investigadores de las universida- 
des tienen su propia definición adecuada a sus intereses individuales".

A pesar de tratarse de un fenómeno bien definido denominado comportamiento autolesivo, Tate y Baroff (1966) lo definieron hace años como el comportamiento según el cual una persona se produce lesiones físicas a sí mismo. Posteriormente, Baumeister y Rollings (1976) lo definieron como aquellos comportamientos o actos que repetidos con frecuencia y que provocan el daño directo a una persona. Como ha sido mencionado anteriormente, el comportamiento autolesivo ha sido definido de una forma muy amplia, por ejemplo, el llamado Global SIB, que es un subtipo específico de autolesión ajustada a unos criterios específicos de daño tisular. Jacobson (1982) definía el SIB como "una acción autolesiva" que impedía al enfermo actuar de forma independiente. Kebbon y Windahl (1985) lo definían como un "movimiento motor evidente, mediante el cual una parte del cuerpo se mueve contra otra parte del cuerpo o contra otros objetos". Incluye comportamientos como golpearse la cabeza, golpearse otras partes del cuerpo, tirarse del pelo, excluyendo conductas como la pica y la provocación de vómitos.

En tercer lugar, definir el nivel de gravedad con el que se clasifica a una persona con problemas autolesivos, de agresividad o de destrucción de la propiedad, es tan arbitrario como crear las definiciones. También podríamos preguntarnos con qué frecuencia debería ocurrir y cuan recientemente debería haber ocurrido con el fin de ser considerado como tal (Borthwick-Duffy 1994).

Los resultados de la correlación sugieren que existe una relación entre el comportamiento agresivo y los desórdenes psiquiátricos, una afirmación que confirman anteriores estudios ya publicados. Por otro lado, también se descubrió que existían más rela- ciones entre los comportamientos agresivos y los trastornos psiquiátricos en los enfermos que vivían en residencias en la comunidad que en otro tipo de enfermos. Citemos la investigación llevada a cabo por Kiernan y Qureshi, en la que se mostró como la esquizofrenia era un rasgo que se encontraba asociado al comportamiento agresivo, seguida por el trastorno afectivo. Los resultados fueron sorprendentes, ya que esperábamos encontrar más trastornos psiquiátricos en los enfermos del hospital que en entre los de la comunidad. Los resultados también reflejaban rasgos clínicos específicos para cada unos de los tres grupos de enfermos. Mientras que el trastorno afectivo y el trastorno adaptativo eran factores comunes que aparecían asociados al comportamiento agresivo de los enfermos que residían con las familias, el trastorno somatoforme y el trastorno de personalidad eran factores comunes asociados al comportamiento agresivo. Por su parte, la esquizofrenia no suponía problema entre los enfermos residentes en hospitales y en familias. Sin embargo, un rasgo clínico muy común entre los enfermos pertenecientes a cada uno de los tres grupos, era el trastorno psicosexual y el trastorno de ansiedad, los cuales no parecían estar asociados al comportamiento agresivo.

De todas maneras, uno debe ser cauto a la hora de interpretar sus descubrimientos sobre Psicopatología. La diferencia entre el comportamiento desafiante o agresivo y los trastornos psiquiátricos no siempre es fácil de identificar. Es frecuente que la investigación incluya cierta preponderancia de personas con graves o profundas discapacidades para el aprendizaje y defina al "trastorno psiquiátrico" incluyendo en él los comportamientos agresivos.

Además, es necesario ser consciente de los problemas de validación que pueden estar incluidos en la PIMRA. Ítems como 
"tiene un extraño lenguaje que resulta disgresivo, vago y sobrelaborado", "sin contactos sociales" y "una conducta peculiar" no diferencian entre una psicosis esquizofrénica y el síndrome de Asperger. Ítems como "ideas delirantes extrañas" requieren una atención especial y suficiente experiencia clínica con el fin de poder diferenciarlas de creencias poco frecuentes que están sancionadas culturalmente y que surgen a de la incomprensión o por falta de inteligencia o simplemente están relacionadas con una cuestión poco usual. Los medios utilizados para el diagnóstico, como la ICD-10 podrían servir de herramienta de comprobación. Varios estudios han descubierto la poca correspondencia existente con el diagnóstico DSM-III, a partir del cual se desarrolló el PIMRA (Maston et al. 1988).

\section{Bibliografía}

AMAN, M.G., SINGH, N.N., STEWART, A.W., FIELD, C.J. Psychometric characteristics of the aberrant behaviour checklist. American Journal of Mental Deficiency, 89, 492502, 1985(a).

AMAN, M.G., SINGH, N.N., STEWART, A.W., FIELD, C.J. The Aberrant Behaviour Checklist: a behaviour rating scale for the assessment of treatment effects. American Journal of Mental Deficiency, 89, 485-491, 1985(b).

BALLINGER, B.R., REID, A.H. Psychiatric disorders in an adult training centre and a hospital for the mentally handicapped, Psychological Medicine, 7, 525-528, 1977.

BAUMEISTER, A.A., ROLLINGS, J.P. Self-injurious behaviour. In Ellis, N.R. (Ed.). International Review of Research in Mental Retardation (Vol. 8, pp. 1-34). New York: Academic Press, 1976.

BORTHWICK-DUFFY, S.A. Prevalence of destructive behaviours: a study of aggression, self-injury, and property destruction. In Thompson, T. Gray, D.B. (Ed.). Destructive Behaviour in Developmental Disabilities. London: SAGE, 1994.
BORTHWICK-DUFFY, S.A., EYMAN, R.K. Who are the dually diagnosed? American Journal on Mental Retardation, 94, 586-595, 1990.

BRUININKS, R.H., OLSON, K.M., LARSON, S.A., LAKIN, K.C. Challenging behaviours among persons with mental retardation in residential settings: implications for policy, research, and practice. In Thompson, T., Gray, D.B. (Ed.). Destructive Behaviour in Developmental Disabilities. London: SAGE, 1994.

BRUININKS, R.H., HILL, B.K., MORREAU, L.E. Prevalence and implications of maladaptive behaviours and dual diagnosis in residential and other service programmes. In Stark, J.A., Menolascino, F.J., Albarelli, M.H., Gray, V.C. (Eds.). Mental Retardation and Mental Health: Classification, Diagnosis, Treatment, Services (pp. 3-29). New York: Springer Verlag, 1988.

CHAMBERLAIN, L., CHUNG, M.C., JENNER, L. Preliminary findings on communication and challenging behaviour in learning difficulty. The British Journal of Developmental Disabilities, XXXIX, 77, 118-125, 1993.

CHUNG, M.C., JENNER, L., CHAMBERLAIN, L., CORBETT, J. One year follow up pilot study on communication skill and challenging behaviour. European Journal of Psychiatry, 9 (2), 83-95, 1995.

CLARE, I.C.H., MURPHY, G.H. MIET (mental impairment evaluation and treatment service): a service option for people with mild mental handicaps and challenging behaviour and/or psychiatric problems. Mental Handicap Research, 6 (1), 70-91, 1993.

CLARKE, D.J., KELLEY, S., THINN, K., CORBETT, J.A. Psychotropic drugs and mental retardation: 1. Disabilities and the prescription of drugs for behaviour and for Epilepsy in three residential settings. Journal of Mental Deficiency Research, 34, 385-395, 1990.

CONNEALLY, S., BOYLE, G., SMYTH, F. An evaluation of the use of small group homes for adults with a severe and profound mental handicap. Mental Handicap Research, 5 (2), 146-168, 1992.

CONROY, J.W., BRADLEY, V.J. The Pennhurst Longitudinal Study: A Report of Five Years of Research and Analysis. Philadelphia: Temple University Developmental Disabilities Center, 1985.

CONROY, J.W., LEMANOWICZ, J.A., FEINSTEIN, C.S., BERNOTSKY, J.M. Results of the CARC V. Thorne Longitudinal Study. Narberth, PA: Conroy and Feinstein Associates, 1991.

CORBETT, J.A. Psychiatric Morbidity and Mental Retardation. In James, F.E., Snaith, R.P. (eds.). Psychiatric Illness and Mental Handicap. London: Gaskell Press, 1979. 
EMERSON, E. Self-injurious behaviour: an overview of recent trends in epidemiological and behavioural research. Mental Handicap Research, 5, 49-81, 1992.

FELCE, D., LOWE, K., PAIVA, S.D. Ordinary housing for people with severe learning disabilities and challenging behaviours. In Emerson, E., McGill, P., Mansell, J. (Ed). Severe Learning Disabilities and Challenging Behaviour: Designing Quality Services. London: Chapman \& Hall, 1994.

FOVEL, J.T., LASH, P.S., BARRON, J.R., D.A., ROBERTS, M.S. A survey of self-restraint, self-injury, and other maladaptive behaviours in an institutionalized retarded population. Research Developmental Disabilities, 10, 377-382, 1989.

GRIFFIN, J.C., WILLIAMS, D.E., STARK, M.T., ALTMEYER, B.K., MASON, M. Self-injurious behaviour: A state-wide prevalence survey of the extent and circumstances. Applied Research in Mental Retardation 7, 105$116,1986$.

HILL, B.K., BRUININKS, R.H. Maladaptive behaviour of mentally retarded individuals in residential facilities. American Journal of Mental Deficiency, 88, 380-387, 1984.

HOEFKENS, A., ALLEN, D. Evaluation of a special behaviour unit for people with mental handicaps and challenging behaviour. Journal of Mental Deficiency Research, 34, 213-228, 1990.

HORNER, R.H., STONER, S.K., FERGUSON, D.L. An Activity-Based Analysis of Deinstitutionalization: The Effects of Community Re-Entry on the Lives of Residents Leaving Oregon's Fairview Training Centre. Eugene: University of Oregon, 1988.

JACOBSON, J.W. Problem behaviour and psychiatric impairment within a developmentally disabled population I: Behaviour frequency. Applied Research in Mental Retardation, 3, 121-139, 1982.

KEBBON, L., WINDAHL, S. Self-injurious behaviour: results of a nation-wide survey among mentally retarded in Sweden. Paper presented at the $7^{\text {th }}$ World Congress of the IASSMD. New Delhi, India, 1985.

KIERNAN, C., QURESHI, H. Challenging Behaviour. In Kiernan, C. (Ed.). Research to Practice? Implications of Research on the Challenging Behaviour of People with Learning Disability. Kidderminster: BILD, 1993.

LAKIN, K.C., HILL, B.K., CHEN, T.H., STEPHENS, S.A. Persons with Mental Retardation and Related Conditions in Mental Retardation Facilities: Selected Findings from the 1987 National Medical Expenditure Survey (Report n. ${ }^{\circ}$ 29). Minneapolis: University of Minnesota, 1989.
LAKIN, K.C., BLAKE, E.M., PROUTY, R.W., MANGAN, T., BRUININKS, R.H. Residential Services for Persons with Developmental Disabilities: Status and Trends Through 1991. Minneapolis: University of Minnesota, 1993.

LARSON, S.A. LAKIN, K.C. Deinstitutionalization of persons with mental retardation: Behavioural outcomes. Journal of the Association for Persons with Severe Handicaps, 14, 324-332, 1989.

LECK, I., GORDON, W.L., McKEOWN, T. Medical and social needs of mentally subnormal patients. British Journal of Preventative and Social Medicine, 21, 115-121, 1967.

LOWE, K., PAIVA, D., FELCE, D. Effects of a community-based service on adaptive and maladaptive behaviours: a longitudinal study. Journal of Intellectual Disability Research, 37, 3-22, 1993.

LUND, J. The prevalence of psychiatric morbidity in mentally retarded adults. Acta Psychiatrica Scandinavica, 72, 563-570, 1985.

MANSELL, J., BEASLEY, F. Small staffed houses for people with a severe learning disability and challenging behaviour. British Journal of Social Work, 23, 329-344, 1993.

MANSELL. J., McGILL, P., EMERSON, E. Conceptualising Service Provision. In Emerson, E., McGill, P., Mansell, J. (Ed.). Severe Learning Disabilities and Challenging Behaviours: Developing High Quality Services. London: Chapman \& Hall, 1992.

MATSON, J.L. The Psychopathology Inventory for Mentally Retarded Adults Manual. Louisiana: International Diagnostic Systems, 1988.

MAURICE, P., TRUDEL, G. Self-injurious behaviour prevalence and relationships to environmental events. In Hollis, J.H., Meyers, C.E. (Eds.). Life Threatening Behaviour. Analysis and Intervention (AAMD Monograph n. ${ }^{\circ} 5$ pp. 81-103). Washington, D.C: American Association on Mental Deficiency, 1982.

MOLONY, H., TAPLIN, J. Deinstitutionalization of people with developmental disability. Australia and New Zealand Journal of Developmental Disabilities, 14, 109. 122, 1988.

MURPHY, G., HOLLAND, A., FOWLER, P., REEP, J. MIETS: a service option for people with mild mental handicaps and challenging behaviour or psychiatric problems. Mental Handicap Research, 4 (1), 41-66, 1985.

OLIVER, C., MURPHY, G.H., CORBETT, J.A. A selfinjurious behaviour in people with mental handicap: a total 
population study. Journal of Mental Deficiency Research, 31, 147-162, 1987.

PRIMROSE, D.A. A survey of 502 consecutive admissions to a subnormality hospital from the $1^{\text {st }}$ January, 1968 to $31^{\text {st }}$ December, 1970. British Journal of Mental Subnormality, 17, 25-28, 1971.

QURESHI, H., ALBORZ, A. Epidemiology of challenging behaviour. Mental Handicap Research, 5, 130$145,1992$.

QURESHI, H. (10. The Size of Problem), Emerson, E., McGill, P., Mansell, J. (Ed). Severe Learning Disabilities and Challenging Behaviour: Designing Quality Services. London: Chapman \& Hall, 1994.

ROJAHN, J. Self-injurious and stereotyped behaviour in noninstitutionalized mentally retarded handicap: Prevalence and classification. American Journal of Mental Deficiency, 91, 268-276, 1986.

RUSSELL, O, HARRIS, P. Assessing the prevalence of aggressive behaviour and the effectiveness of interventions. In Kiernan, C. (Ed). Research to Practice? Implications of Research on the Challenging Behaviour of People with Learning Disability. Kidderminster. BILD, 1993.
SCHROEDER, S.R., SCHROEDER, C.S., SMITH, B., DALLDORF, J. Prevalence of self-injurious behaviour in a large state facility for the retarded: A three-year follow-up study. Journal of Autism and Childhood Schizophrenia, 8, 261-169, 1978.

TATE, B. G., BAROFF, G. S. Aversive control of selfinjurious behaviour in a psychotic boy. Behaviour Research and Therapy, 4, 281-287, 1966.

WILLIAMS, C.E. A study of patients in a group of mental subnormality hospitals. British Journal of Mental Subnormality, 17, 29-41, 1971.

WING, L. Hospital Closure and the Resettlement of Residents. Aldershot: Avebury, 1989.

Dirección para correspondencia:

Man Cheung Chung

University of Plymouth

Department of Psychology

Drake Circus

Plymouth

PL4 8AA

REINO UNIDO 